\title{
PARADIGM-HF Trial and its Important Clinical Meanings
}

\author{
Jin-lai Liu* \\ Department of cardiology, The Third Affiliated Hospital, SUN Yat-sun University, Guangzhou 510630, China \\ *Corresponding author: Jin-lai Liu, Department of cardiology, The Third Affiliated Hospital, SUN Yat-sun University, Guangzhou 510630, China, E-mail: \\ lj.lai@medmail.com.cn
}

Received date: May 26, 2015; Accepted date: June 23, 2015; Published date: June 25, 2015

Copyright: @ 2015 Liu JL. This is an open-access article distributed under the terms of the Creative Commons Attribution License, which permits unrestricted use, distribution, and reproduction in any medium, provided the original author and source are credited.

\begin{abstract}
ACEi/ARB and $B$ blocker and aldosterone antagonist have been a gold standard for treating chronic heart failure with reduced ejection fraction (HFrEF). The paper described simply the PARADIGM-HF study (Prospective comparison of ARNi with ACEi to Determine Impact on Global Mortality and morbidity in HF trial), and the results showed that compared to enalapril, LCZ69(ARNi) significantly reduced the primary endpoint (cardiovascular death or hospitalization for HF) [HR0.80 (95\% Cl 0.73-0.87), $\mathrm{P}<0.001$; NNT 21], cardiovascular death [HR0.80 (95\% Cl 0.71-0.89), $\mathrm{P}<0.001$; NNT 31], hospitalization for HF [HR0.79 (95\% Cl 0.71-0.89), $P<0.001$; NNT 36], and death from any cause [HR0.84 $(95 \% \mathrm{Cl} 0.76-0.93), \mathrm{P}<0.001$; NNT 36]. LCZ696 was well tolerated. The PARADIGM-HF study has an important clinical significance and would change the pattern of HF treatment in the future.
\end{abstract}

Keywords: PARADIGM-HF trial; Chronic heart failure with reduced ejection fraction (HFrEF); Angiotensin receptor-neprilysin inhibitor (ARNi)

\section{Commentary}

Heart failure (HF) is a complex clinical syndrome that results from any structural or functional impairment of ventricular filing or ejection of blood. HF is divided as HF with preserved EF (HFpEF) and HF with reduced EF (HFrEF). HFrEF is defined as the clinical diagnosis of $\mathrm{HF}$ and $\mathrm{EF} \leq 40 \%$, and $\mathrm{HFpEF}$ criteria include as a) clinical signs or symptoms of HF; b) evidence of preserved or normal LVEF; and c) evidence of abnormal LV diastolic dysfunction that can be determined by Doppler echocardiography or cardiac catheterization. According to the exercise capacity and the symptomatic status of HF, the New York Heart Association (NYHA) functional classification is used. Angiotensin converting enzyme inhibitor (ACEi)/angiotensin receptor blocker (ARB) and $B$ blocker and aldosterone antagonist have been a gold standard for treating HFrEF.

However, on August 30, 2014, the results of the PARADIGM-HF (Prospective comparison of ARNi with ACEi to determine impact on global mortality and morbidity in heart failure trial) trial were released at the European Society of Cardiology, and was also published in the New England Journal of Medicine at the same time [1]. PARADIGMHF trial compared a new angiotensin receptor antagonist-neprilysin inhibitor LCZ696 (ARNi) with angiotensin converting enzyme inhibitor enalapril (ACEi) in reducing mortality in patients with chronic heart failure with reduced ejection fraction (HFrEF). The trial was stopped early, and the result showed that as compared with enalapril, LCZ696 significantly reduced the risks of the composite primary endpoint, death from cardiovascular causes, death from any cause and hospitalization for heart failure. PARADIGM-HF trial has an important meaning because it maybe changes the pattern of $\mathrm{HF}$ treatment in the future.

\section{PARADIGM-HF trial brief introduction}

In this double-blind trial, 8,442 patients with class II, III, or IV heart failure and an ejection fraction of $40 \%$ or less were randomized from 947 sites in 47 countries, and the trial was stopped early after a median follow up of 27 months [1]. Of these, 43 patients were removed from final analysis because of misrandomization or major good clinical practice violations at the sites. Ultimately, 8,399 patients were analyzed. Inclusion criteria included: left ventricular ejection fraction $(\mathrm{LVEF}) \leq 35 \%$; plasma B-type natriuretic peptide (BNP) $\geq 150 \mathrm{pg} / \mathrm{ml}$ (or an N-terminal pro-BNP [NT-proBNP] $\geq 600 \mathrm{pg} / \mathrm{ml}$ ) or, if they had been hospitalized for heart failure within the previous 12 months, a $\mathrm{BNP} \geq 100 \mathrm{pg} / \mathrm{ml}$ (or an NT-proBNP $\geq 400 \mathrm{pg} / \mathrm{ml}$ ); to take a stable dose of a beta-blocker and an ACE inhibitor (or ARB) equivalent to at least $10 \mathrm{mg}$ of enalapril daily. Exclusion criteria: symptomatic hypotension $(\mathrm{SBP}<95 \mathrm{mmHg})$; estimated glomerular filtration rate (eGFR) $<30 \mathrm{ml} / \mathrm{min} / 1.73 \mathrm{~m}^{2}$; serum potassium $>5.2 \mathrm{mmol} / \mathrm{l}$; history of angioedema or unacceptable side effects during receipt of ACE inhibitors or ARBs. Patients were randomly assigned in the enalapril group (10 mg twice daily) or the LCZ696 group (200 mg twice daily). The results showed that as compared with enalapril, LCZ696 significantly reduced the primary outcome [HR0.80 (95\% CI $0.73-0.87) \mathrm{p}<0.001 ; \mathrm{NNT} 21]$, death from cardiovascular causes [HR0.80 (95\% CI 0.71- 0.89)p $<0.001$; NNT 31], hospitalization for heart failure [HR0.79 (95\% CI 0.7,1- 0.89)p $<0.001$; NNT36], and death from any cause [HR0.84 (95\% CI 0.76-0.93)p $<0.001$; NNT 36], and also improved the symptoms and physical limitations of heart failure. PARADIGM-HF trial has shown not only the significant clinical efficacy, but also a good safety. Adverse reactions included hypotension [14\% in the LCZ696 group vs. $9.2 \%$ in the enalapril group $(\mathrm{p}<0.001)]$, nonserious angioedema $(0.2 \%$ vs. $0.1 \%)$, renal impairment ( $3.3 \%$ vs. $4.5 \%, \mathrm{p}=0.007)$, hyperkalemia $(16.1 \%$ vs. $17.3 \%)$, and cough (11.3\% vs. $14.3 \%, \mathrm{p}<0.001)$.

\section{The clinical significance of PARADIGM-HF trial}

For nearly 25 years, ACEi has been a cornerstone for treating EFrHF. Long-term treatment with enalapril decreased the relative risk 
of death by $16 \%$ (95\% CI $5 \%-26 \%$; NNT 22 ) among patients with mild-to-moderate symptoms [2]. The use of beta-blockers and mineralocorticoid- receptor antagonists, when added to ACEis, resulted in incremental decreases in the risk of death of 34\% (95\% CI $19 \%-47 \%$; NNT 26) and 30\% (95\% CI 18\%-40\%; NNT 9), respectively $[3,4]$. The guidelines in many countries put ACEi/ARB and betablocker and mineralo-corticoid receptor antagonists as golden standard for the treatment of EFrHF. Neprilysin, a neutral endopeptidase, degrades several endogenous vasoactive peptides, including natriuretic peptides, bradykinin, and adrenomedullin. Inhibition of neprilysin counters the neurohormonal over-activation that contributes to vasoconstriction, sodium retention, and maladaptive remodeling. LCZ696, which consists of the neprilysin inhibitor prodrug sacubitril (AHU377) which is converted enzymatically to the active neprilysin inhibitor LBQ657, and valsartan, is the first of a new class of drugs that simultaneously block the reninangiotensin-aldosterone system (RAAS), and the natriuretic peptide system (NPS). LCZ696 blocks RAAS activation while enhancing the adaptive actions of atrial natriuretic peptide (ANP). The magnitude of the benefit of LCZ696 over ACEi was highly significant and up to $20 \%$ on the base of ACEi 16\% benefit and LCZ696 had a good safety. The recent meta-analysis showed that compared with ACEi or ARB alone, combined NEP-RAAS inhibition resulted in a reduction in risk of decline in renal function (risk ratio $0.68,95 \%$ CI $0.51-0.92, \mathrm{p}=0.01$ ) [5]. Therefore, dual inhibition of the renin-angiotensin-aldosterone system and neprilysin inhibition represent a novel approach to treating patients with HF. We maybe predict that the results of more clinical trials will challenge the standard treatment on HFrEF at present. LCZ696 could replace ACEi and ARBs as first-line therapy in the treatment of patients with HFrEF [6].

\section{The Future}

Given that PARADIGM-HF's encouraging results, how the next researches are? First of all, the effect of LCZ696 on in patients with chronic heart failure with preserved ejection fraction (HFpEF) [7]. The PARAMOUNT study served as a hypothesis-generating trial for HFpEF. PARAMOUNT trial showed that comparing with the valsartan group, LCZ696 significantly reduced NT-proBNP (605 $\mathrm{pg} / \mathrm{ml}$ vs $835 \mathrm{pg} / \mathrm{ml}, \mathrm{p}=0.005)$ at 12 weeks. At present the larger scale PARAGON (Prospective Comparison of LCZ696 with ARB Global Outcome in HF with Preserved Ejection Fraction) trial (NCT01920711) has begun. Second, aortic stiffness- in the Strong Heart study, central systolic pressure was a predictor of left ventricular hypertrophy, whereas pulse pressure was a predictor of vascular hypertrophy [8,9]. The evidence showed that LCZ696 reduced both ambulatory systolic and pulse pressures more than did valsartan. So, the PARAMETER (Prospective comparison of Angiotensin Receptor neprilysin inhibitor with Angiotensin receptor blocker Measuring arterial stiffness in the Elderly) study (NCT01692301) is comparing LCZ696 with olmesartan in elderly hypertensive patients with a pulse pressure $>60 \mathrm{~mm} \mathrm{Hg}$. The endpoints are changes in central aortic systolic and pulse pressures determined noninvasively. Third, renal disease- the evidence shows that RAAS inhibitor slows progression in patients with chronic kidney disease, with and without diabetes. There is being tested prospectively in the UKHARP (UK Heart and Renal Protection) trial, which is comparing LCZ696 with irbesartan in the efficacy and safety, in patients with proteinuric renal disease and an estimated glomerular filtration rate (eGFR) $20-60 \mathrm{ml} / \mathrm{min} / 1.73 \mathrm{~m} 2$. LCZ696 is also promising for the treatment of serious and/or resistant hypertension.

\section{Conclusion}

In conclusions, the PARADIGM-HF study has an important clinical significance and would change the pattern of HF treatment in the future. LCZ696 may replace conventional RAAS inhibitors in many patients with chronic HFrEF in our clinical practice. The potential value of LCZ696 in HFpEF, in acute HF, in HF patients with the cardiorenal syndrome, in the prevention of HF in asymptomatic patients with left ventricular hypertrophy, dilation, and/or dysfunction and in severe hypertension remains to be determined. ARNi will show a vast clinical prospect.

\section{References}

1. McMurray JJV, Packer M, Desai AS, Gong J, Lefkowitz MP, et al. (2014) Angiotensin-neprilysin inhibition versus enalapril in heart failure. $\mathrm{N}$ Engl J Med 371: 993-1004.

2. The SOLVD Investigators (1991) Effect of enalapril on survival in patients with reduced left ventricular ejection fractions and congestive heart failure. N Engl J Med 325: 293-302.

3. MERIT-HF Study Group (1999) Effect of metoprolol CR/XL in chronic heart failure: Metoprolol CR/XL Randomised Intervention Trial in Congestive Heart Failure (MERIT-HF). Lancet 353: 2001-2007.

4. Pitt B, Zannad F, Remme WJ, Cody R, Castaigne A, et al. (1999) The effect of spironolactone on morbidity and mortality in patients with severe heart failure. N Engl J Med 341:709-717.

5. Bodey F, Hopper I, Krum H (2015) Neprilysin inhibitors preserve renal function in heart failure. Int J Cardiol 179: 329-330.

6. Vardeny O, Miller R, Solomon SD (2014) Combined neprilysin and renin-angiotensin system inhibition for the treatment of heart failure. JACC Heart Fail 2: 663-670.

7. Braunwald E (2015) The path to an angiotensin receptor antagonistneprilysin inhibitor in the treatment of heart failure. J Am Coll Cardiol 65: 1029-1041.

8. Roman MJ, Okin PM, Kizer JR, Lee ET, Howard BV, et al. Relations of central and brachial blood pressure to left ventricular hypertrophy and geometry: the Strong Heart Study. J Hypertens 28: 384-388.

9. Ruilope LM, Dukat A, Bohm M, Lacourcière Y, Gong J, et al. Blood pressure reduction with LCZ696, a novel dual acting inhibitor of the angiotensin II receptor and neprilysin: a randomised, double-blind, placebo controlled, active comparator study. Lancet 375: 1255-1266. 